\title{
ADAPTIVE MULTIRESOLUTION DECOMPOSITION: APPLICATION TO LOSSLESS IMAGE COMPRESSION
}

\author{
Hocine Bekkouche and Michel Barret \\ Supélec, Équipe Signaux et Systèmes Electroniques \\ 2 rue É. Belin 57070 METZ FRANCE \\ Hocine.Bekkouche@supelec.fr and Michel.Barret@supelec.fr
}

\begin{abstract}
In this paper we introduce the use of adaptive filter banks in lossless compression of images with progressive coding in resolution. During the decomposition the filter adapts itself automatically to various regions of the image, preserving the perfect reconstruction property. Effects of parameters used in the decomposition have been studied. Simulation results are given and compared with well-known codecs. The proposed scheme gives, on average, smaller lossless compression bit rate. However, This improvement in performance is achieved at the expense of an increase in computational complexity.
\end{abstract}

\section{INTRODUCTION}

The lossless compression of images finds applications in satellite and medical imaging, where a near lossless coding is not satisfactory. The techniques of progressive coding, like EZW algorithm of Shapiro [1] or SPIHT algorithm introduced by Said and Pearlman [2], are based on multiresolution decompositions of the initial image and they are widely used today for near lossless or lossless image coding [3]. In these multiresolution decompositions, the coefficients of the filters are selected among a predetermined set of possible values, but they are not calculated in order to adapt at best to the image. In addition, most of the lossless image compression algorithms used today, like CALIC [4] and LOCO-I [5], are not associated with multi-resolution transformations. On the other hand, they adapt themselves to the image using context-based predictors.

Over the last years, image compression algorithms using the linear mean squared estimation (LMSE) techniques have been tested (see [6] and its bibliography), but they were not associated with pyramidal hierarchical decompositions. Recently, lossless image compression techniques combining the LMSE and the pyramidal hierarchical decomposition have appeared. Some of them use wavelets decom-

Published in Proc. ICASSP, Orlando, Florida, USA, May 2002 This work was supported in part by the Lorraine Region. positions [7, 8], while others use subband decompositions with nonlinear filters [9]. An adaptive polyphase subband decomposition structure, based on the LMS algorithm, was proposed in [10]. We presented in [11] a multiresolution decomposition method with a global adaptation. Although this adaptation provides an optimal prediction filter (from least squares point of view), it is still impossible to track abrupt changes of local statistics in the image.

In this paper we present a multiresolution decomposition technique based on the recursive least-squares (RLS) algorithm. An important feature of the RLS algorithm is that it converges faster than the LMS algorithm, which makes it more effective for nonstationary signals, such as images. Moreover, the use of an ARMA model provides a better estimation than an AR model, which improves the compression performance. Progressive coding in resolution is possible with the proposed scheme. However, it does not permit progressive coding in quality, because the decoder cannot recover the filter coefficients from the truncated bit stream. That leads to a rapid divergence of the algorithm.

In the second section, the proposed perfect reconstruction adaptive multiresolution decomposition is described in details. In section 3, effects of parameters are experimentally studied and a comparisons with well-known codecs as SPIHT [2], LOCO-I (JPEG-LS) [5] and CALIC [4] is presented.

\section{MULTIRESOLUTION DECOMPOSITIONS}

The general principle of the subband decompositions we have implemented is shown in the block diagram of Fig. 1. The original image $I$ is decomposed by decimation into two sub-images $I_{1}$ and $I_{2}$.

The image $I_{2}$ is estimated according to the linear relation:

$$
\begin{aligned}
\hat{I}_{2}(i, j)= & \sum_{(h, k) \in \Delta_{1}} a_{h k} I_{1}(i-h, j-k) \\
& +\sum_{(h, k) \in \Delta_{2}} b_{h k} I_{2}(i-h, j-k),
\end{aligned}
$$




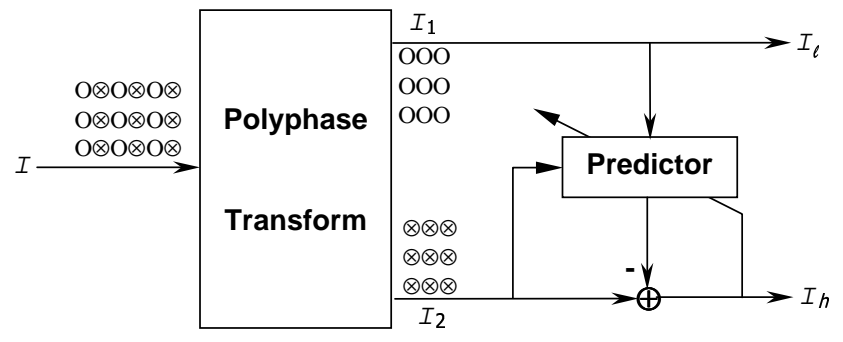

Fig. 1. Subband decomposition. The original image $I$ is decomposed into two sub-images $I_{\ell}$ and $I_{h}$.

where $\Delta_{1}$ and $\Delta_{2}$ are finite subsets of $\mathbb{Z}^{2}$. The coefficients $a_{h k}$ and $b_{h k}$ are updated during the decomposition by the RLS method. The estimated image $I_{2}$ is scanned in a raster manner. Initially the original image $I$ is replaced by two sub-images $I_{\ell}$ and $I_{h}$ defined by the equations:

$$
\begin{aligned}
I_{\ell} & =I_{1} \\
I_{h}(i, j) & =I_{2}(i, j)-\left\lfloor\hat{I}_{2}(i, j)\right\rfloor,
\end{aligned}
$$

where for a real number $x,\lfloor x\rfloor$ represents the integer part of $x+1 / 2$. Thereafter we apply again the same process (exchanging lines and columns) on the sub-image $I_{\ell}$ in order to obtain the two sub-images $I_{\ell \ell}$ and $I_{\ell h}$. The other subimage $I_{h}$ in only subject to the polyphase transform, giving the sub-images $I_{h \ell}$ and $I_{h h}$. Hence one level of decomposition transforms $I$ into four sub-images $I_{\ell \ell}, I_{\ell h}, I_{h \ell}$ and $I_{h h}$. Finally, for the multiresolution decomposition, we apply the same process to the low resolution $I_{\ell \ell}$ image. Let $\Delta_{1}(p)$ be the set of relative integer couples whose absolute value of each term is less than or equal to $p$, and let

$$
\Delta_{2}(p)=\left\{(h, k) \in \mathbb{Z}^{2} \mid \begin{array}{lll}
h=0 & \text { and } & 1 \leq k \leq p \\
|k| \leq p & \text { and } \quad 1 \leq h \leq p
\end{array}\right\}
$$

Let us introduce the vector $y_{1}(i, j)$ (resp. $y_{2}(i, j)$ ) of dimension $r_{1}$ (resp. $r_{2}$ ) containing the pixels $I_{1}(i-h, j-$ $k)\left.\right|_{(h, k) \in \Delta_{1}(p)}\left(\right.$ resp. $\left.\left.I_{2}(i-h, j-k)\right|_{(h, k) \in \Delta_{2}(p)}\right)$, reordered as indicated in figure 2(a) (resp. in figure 2(b)) and where only the $r_{1}$ (resp. $r_{2}$ ) first elements are retained. With this reordering, only the $r_{1}+r_{2}$ closest pixels to $I(i, j)$ appear in the observation vector $\underline{y}(i, j)=\left(\underline{y}_{1}^{t}(i, j), \underline{y}_{2}^{t}(i, j)\right)^{t}$. The subsets $\Delta_{1}$ and $\Delta_{2}$ permit a perfect reconstruction in decoding. Using the coefficients $a_{h k}$ and $b_{h k}$, the vector $c(i, j)$ of the estimation filter coefficients is built in the same manner as the observation vector. In terms of these matrices definitions, equation (1) is written as follows:

$$
\hat{I}_{2}(i, j)=\underline{c}^{t}(i, j) \underline{y}(i, j)
$$

The optimal filter $\underline{\hat{c}}^{t}(i, j)$, from the least squares point of view, is the solution of the system of normal equations [12] and is given by:

$$
\underline{c}^{t}(i, j)=\boldsymbol{\Phi}^{-1}(i, j) \underline{\theta}
$$

where $\boldsymbol{\Phi}$ is the autocorrelation matrix of the observation vector and $\underline{\theta}$ is the cross-correlation vector between the observation vector and the desired response $I_{2}(i, j)$. This direct solution needs the inversion of the autocorrelation ma-

\begin{tabular}{|c|c|c|c|c|c|c|}
\hline \multicolumn{7}{|c|}{$4 h$} \\
\hline 49 & 44 & 36 & 28 & 35 & 43 & 48 \\
\hline 45 & 24 & 18 & $1 \mid$ & 17 & 23 & 42 \\
\hline 37 & 19 & 7 & 2 & 6 & 16 & 34 \\
\hline 29 & 12 & 3 & 1 & 5 & 10 & 27 \\
\hline 30 & 20 & 8 & 4 & 9 & 15 & 33 \\
\hline 38 & 25 & 21 & 13 & 14 & 22 & 41 \\
\hline 46 & 39 & 31 & 26 & 32 & 40 & 47 \\
\hline
\end{tabular}
trix. To avoid performing such an operation we calculate for each $(i, j)$ the least square estimate $\underline{c}(i, j)$ recursively. The adaptive decomposition algorithm is summarized in table 1 .

(a)

Fig. 2. Order of appearance of the pixels for $p \leq 3$ in (a) $\Delta_{1}(p)$ and $(b) \Delta_{2}(p)$.

\section{PRINCIPAL RESULTS}

We chose a set of 8-bits test images, with various sizes and complexities. In order to obtain a complete lossless image compression system we combine the proposed RLS-based adaptive multiresolution decomposition with an arithmetic coder with adaptive model. The use of a forgetting factor $\alpha$ (see Tab. 1) equal to 1 is suited for a stationary environment, for which the best steady-state results are obtained. However, when the RLS algorithm operates in a nonstationary environment such as images, the algorithm must use finite memory. A value of $\alpha$ less than 1 is used. By doing so, the algorithm attains the capability to track statistical variations in the environment in which it operates. An experimental value of the forgetting factor $\alpha$ equal to 0.9995 is a good compromise. This value is used in all our simulations. The results of lossless compression of five images are given in table 2. The $\alpha=0.9995$ case performs significantly better than $\alpha=1$ case for all the five images. To extend the image at boundaries, two techniques are possible: periodic extension and symmetric extension. It is intuitive and has also been verified by our experiments that the symmetrical 
Table 1. Estimation and adaptation algorithm.

$$
\begin{aligned}
& \text { Initialisation } \\
& \underline{c}=(1,0, \ldots, 0)^{t} \text {, with } \underline{c} \in \mathbb{R}^{r_{1}+r_{2}} \\
& \mathbf{K}=\delta^{-1} \mathbf{I} \text {, with } \mathbf{I}, r_{1}+r_{2} \text { order identity matrix } \\
& \text { and } \delta \text { a small positive constant. } \\
& \alpha=0,9995, \text { forgetting factor. } \\
& \text { for } i=1,2, \ldots \\
& \text { for } j=1,2, \ldots \\
& I_{\ell}(i, j)=I_{1}(i, j) \\
& \hat{I}_{2}(i, j)=\left\lfloor\underline{c}^{t} y(i, j)\right\rfloor \\
& I_{h}(i, j)=I_{2}(\bar{i}, j)-\hat{I}_{2}(i, j) \\
& \underline{u}=\mathbf{K} \underline{y}(i, j) \\
& \mathbf{K}=\frac{1}{\alpha}\left(\mathbf{K}-\frac{\underline{u} \underline{u}^{t}}{\alpha+y(i, j)^{t} \underline{u}}\right) \\
& \underline{c}=\underline{c}+I_{h}(i, j) \mathbf{K} \underline{y}(i, j) \\
& \text { end loop } j \\
& \text { end loop } i
\end{aligned}
$$

extension gives better compression results than periodic extension. This is due to the fact that the symmetric extension does not introduce jump discontinuities in the extended signal. Discontinuities are undesirable, because they increase the amount of high frequency energy in the signal. Lossless compression results for the same five test images are shown in table 2. In general, the results obtained with symmetric extension are comparable to those obtained with periodic extension. In figure 3, the lossless compression bit rates are plotted versus $r_{1}$ and $r_{2}$. It can be clearly seen that the use of an ARMA estimation model $\left(r_{2} \neq 0\right)$ gives smaller compression bit rate than an AR model $\left(r_{2}=0\right)$. It can be noticed in figure 3 that the compression bit rate decreases when $r_{1}$ or $r_{2}$ increases. This reduction vanishes when $r_{1}$ and $r_{2}$ reach their optimal values. These optimal values change according to the type of the image. For natural images, $\left(r_{1}, r_{2}\right)=(8,4)$ is a satisfactory choice, while for MRI images, which are generally smooth images, a small number of pixels is sufficient to have a good estimation and the experimentation has shown that the values $\left(r_{1}, r_{2}\right)=(6,0)$ are sufficient. The choice of $r_{1}$ and $r_{2}$ is limited by the computing time. The figure 4 shows the variations of the computing time for an image $512 \times 512$ versus $r_{1}$ (for $r_{2}=1$ ), the adaptive decomposition is computationally demanding. Its computational complexity is $\left.O\left(r_{1}+r_{2}\right)^{2}\right)$. However, its mathematical formulation and therefore the implementation is relatively simple. This computational complexity can be reduced, without loss of performance, to $O\left(r_{1}+r_{2}\right)$ by implementing a fast version of RLS [12]. The table 3 shows a comparative evaluation of the

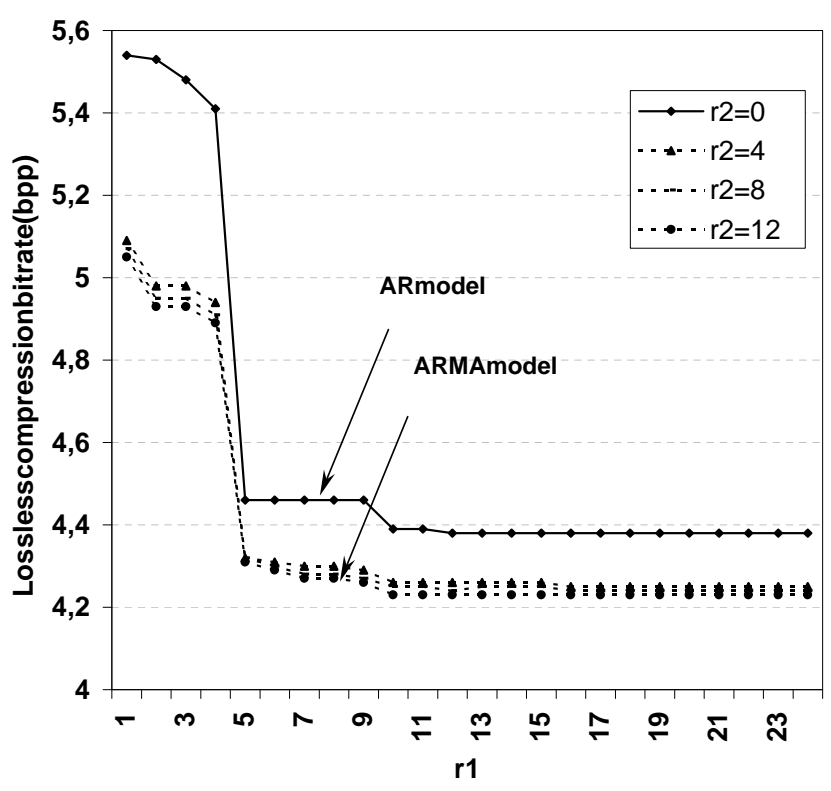

Fig. 3. Lossless compression bit rate in $b p p$ versus filter order, obtained with the image Fingerl (512x512).

\begin{tabular}{|c|c|c|c|}
\hline & P.E. & \multicolumn{2}{|c|}{ S.E. } \\
\hline image & $\alpha=0.9995$ & $\alpha=0.9995$ & $\alpha=1$ \\
\hline Lena & 4.21 & 4.21 & 4.22 \\
\hline Goldhill & 4.72 & 4.71 & 4.74 \\
\hline Barbara & 4.54 & 4.54 & 4.65 \\
\hline Irm1 & 2.89 & 2.88 & 3.18 \\
\hline Finger1 & 4.23 & 4.23 & 4.29 \\
\hline
\end{tabular}

Table 2. Lossless compression results (in $b p p$ ) for periodic extension (P.E.) and symmetric extension (S.E.), and for two values of the forgetting factor, $\alpha=0.9995$ and $\alpha=1$

performance of the proposed RLS-based lossless compression codec with other lossless compression systems such as SPIHT [2], LOCO-I (JPEG-LS) [5] and CALIC [4].

\section{CONCLUSION}

In this paper we have introduced an RLS-based adaptive subband decomposition scheme. The filter adapts itself to different regions of the image, hence it tracks the nonstationarities. We applied this scheme to the lossless image compression. An experimental study and a comparison of compression results with other methods are presented. The proposed compression system gives, on average, smaller compression bit rates than other codecs, with the trade-off of an increase in the computational complexity. 


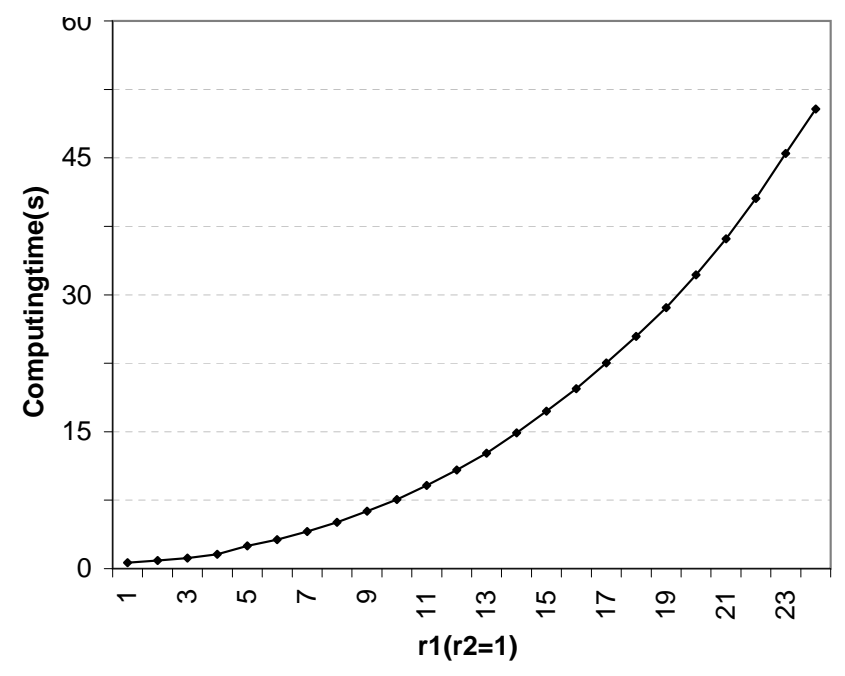

Fig. 4. Computation time versus filter order, obtained for a $512 \times 512$ image.

\section{REFERENCES}

[1] J. M. Shapiro, "Embedded image coding using zerotrees of wavelet coefficients," IEEE Trans. on Signal Processing, vol. 41, no. 12, pp. 3445-3462, Dec. 1993.

[2] A. Said and W. A. Pearlman, "A new, fast and efficient image codec based on Set Partitioning In Hierarchical Trees," IEEE Trans. on Circuits and Systems for Video Technology, vol. 6, no. 3, pp. 243-250, Jun. 1996.

[3] A. Said and W. A. Pearlman, "An image multiresolution representation for lossless and lossy compression," IEEE Trans. on Image Processing, vol. 5, pp. 1303-1310, Sep. 1996.

[4] X. Wu and N. Memon, "Context-based, adaptive, lossless image coding," IEEE Trans. on communications, vol. 45, no. 4, pp. 437-444, Apr. 1997.

[5] M. Weinberger, G. Seroussi, and G. Sapiro, "LOCO-I: A low complexity, context-based, lossless image compression algorithm," Proc. IEEE Data Compression Conference, Mar. 1996.

[6] Y. S. Chung and M. Kanefsky, "On 2D recursive LMS algorithms using ARMA prediction for ADPCM encoding of images," IEEE Trans. on Image Proc., vol. 1, no. 3, pp. 416-422, Jul. 1992.

[7] M. D. Adams and F. Kossentini, "Reversible integerto-integer wavelet transforms for image compression: performance evaluation and analysis," IEEE Trans. on Image Proc., vol. 9, no. 6, pp. 1010-1024, Jun. 2000.

\begin{tabular}{|c|c|c|c|c|}
\hline Image & SPIHT & CALIC & LOCO-I & proposed \\
\hline Lena & 4.17 & 4.20 & 4.25 & 4.24 \\
\hline Goldhill & 4.75 & 4.67 & 4.73 & 4.74 \\
\hline Barbara & 4.56 & 4.63 & 4.75 & 4.51 \\
\hline Mandrill & 5.93 & 5.99 & 6.03 & 5.97 \\
\hline Peppers & 4.54 & 4.47 & 4.50 & 4.59 \\
\hline Irm1 & 3.12 & 3.19 & 3.13 & 2.88 \\
\hline Irm2 & 3.30 & 3.43 & 3.38 & 2.85 \\
\hline Irm3 & 5.05 & 5.24 & 5.26 & 5.02 \\
\hline Finger1 & 4.47 & 4.55 & 4.57 & 4.24 \\
\hline Finger2 & 3.94 & 3.99 & 3.96 & 3.77 \\
\hline Finger3 & 4.46 & 4.51 & 4.52 & 4.24 \\
\hline Texture1 & 6.57 & 6.69 & 6.72 & 6.46 \\
\hline Texture2 & 5.95 & 5.98 & 5.97 & 5.93 \\
\hline Texture3 & 6.09 & 6.02 & 6.06 & 5.96 \\
\hline Airplane & 4.50 & 4.85 & 4.59 & 4.45 \\
\hline Airport & 5.32 & 5.31 & 5.35 & 5.19 \\
\hline \hline Mean & 4.73 & 4.80 & 4.80 & 4.61 \\
\hline
\end{tabular}

Table 3. Comparative evaluation of lossless compression results (in $b p p$ ) obtained with SPIHT, LOCO-I (JPEG-LS), CALIC and the proposed method (with parameters $r_{1}=16$, $r_{2}=8$ and $\left.\alpha=0.9995\right)$.

[8] V. N. Ramaswamy, N. Ranganathan, and K. R. Namuduri, "Performance analysis of wavelets in embedded Zerotree-based lossless image coding schemes," IEEE Trans. on Signal Proc., vol. 47, no. 3, pp. 884889, Mar. 1999.

[9] F. J. Hampson and J. C. Pesquet, "M-band nonlinear subband decomposition with perfect decomposition," IEEE Trans. on Image Proc., vol. 7, no. 11, pp. 15471560, Nov. 1998.

[10] Ö. N. Gerek and A. E. Çetin, "Adaptive polyphase subband decomposition structures for image compression," IEEE Trans. on Image Proc., vol. 9, no. 10, pp. 1649-1660, Oct. 2000.

[11] M. Barret and H. Bekkouche, "Adapted nonlinear multiresolution decomposition with applications in progressive lossless image coding," International Symposium on Image and Signal Processing and Analysis ISPA'01, Pula, Croatia, pp. 609-612, Jun. 2001.

[12] S. Haykin, Adaptive filter theory, Engelwood Cliffs, NJ: Prentice-Hall, second edition, 1991. 